\title{
Criterios decisionales para la resolución de problemas. Un Modelo de gestión del ingeniero industrial
}

\begin{abstract}
RESUMEN
El objetivo del estudio es identificar los criterios que el ingeniero industrial, como decisor o directivo, aplica para resolver situaciones problemáticas, para lo cual se diseña el modelo conceptual de criterio con la metodología de los sistemas blandos y un diseño experimental para validar exploratoriamente la influencia del criterio en la elección del directivo o decisor. Dentro del marco racional empleado en el manejo de organizaciones, se intenta identificar los criterios que el decisor aplica para elegir entre opciones, y resolver las situaciones problemáticas de los diversos escenarios donde actúa y ejerce roles. En la empresa se consideran cuatro tipos de macrocriterios decisionales, relacionados al escenario y a la perspectiva del decisor, éstos pueden ser: a) de posición, basados en la naturaleza humana, b) de optimización, basados en el logro y la acción en el mundo real, c) de satisfacción, basados en la regulación social y la conducta, d) de oportunidad, basados en predicciones y contingencias, El estudio muestra los principales criterios para tomar decisiones y la preferencia del ingeniero industrial por uno $\mathrm{u}$ otro, en función al escenario y al rol que ejerza en el momento de la elección.
\end{abstract}

Palabras clave: Criterio de decisión, Escenario, Toma de decisiones

DECISION CRITERIA FOR PROBLEM

SOLVING. AN INDUSTRIAL

ENGINEER'S MANAGERIAL MODEL

\section{ABSTRACT}

The aim of this study is to identify the criteria that the industrial engineer or manager as decision maker, applied to solve problems, for which you design the conceptual model approach to soft systems methodology and experimental design to validate the influence of criterion in the choice of the manager or decisor maker. Within the rational framework used in the management of organizations, the industrial engineer try to identify the criteria, as decision maker, applied to choose among options, and resolve problem situations of the various scenarios in which it operates and holds roles. The enterprise considers four types of decisional macrocriteria, related to the stage and the decision maker's perspective, they may include: a) Position, based on human nature, b) Optimization based on the achievement and action in the world real, c) Satisfaction, based on social regulation and behavior. d) Opportunity, based on predictions and contingencies. The study shows main criteria to make decisions and industrial engineer's preference for either, depending on the stage and the role that manage at time of election.

Keywords: Decisional Criteria, Scenario, Decision Making

\section{INTRODUCCIÓN}

El presente estudio tiene el objetivo de identificar los criterios que el ingeniero industrial aplica para elegir entre diversas opciones y resolver situaciones problemáticas, en su rol decisor cuando desempeña tareas directivas. Se sustenta en el enfoque de toma de decisiones racionales, decisiones en operaciones, los sistemas de actividad humana y las habilidades de gestión del ingeniero industrial.

\section{Antecedentes filosóficos y académicos}

Criterio significa regla, juicio o decisión. Etimológicamente deriva del griego kpíveıv, que significa juzgar. Criterio es la capacidad de formarse un juicio para tomar una decisión aplicando reglas que se consideran verdaderas, donde lo verdadero es diferenciar lo correcto de lo incorrecto. Criterio es el buen juicio para elegir (4).

Entre los antecedentes de los criterios, se identifican los tres estados, dentro de los cuales las personas se orientan en la vida y toman decisiones, según Kierkegaard (14) cada estado o fase representa una actitud vital. Ortega y Gasset define la razón vital y afirma que, en la filosofía de la acción lo importante son los hechos en el mundo real, sensorial y visible (10).

Buffa (4) plantea que las decisiones se basan en los criterios, el cual tamiza lo adecuado y conveniente. Según Read, el ingeniero industrial asume nuevos roles en la administración de organizaciones tomando decisiones para resolver problemas y reducir las fallas o efectos inesperados o indeseados (15). Los escenarios donde el decisor ejerce roles y toma decisiones son los campos de circunstancia (1) que se basan en la teoría de campos de Lewin (11).

\section{Epistemología de los criterios de decisión}

Los criterios pueden ser objetivos y subjetivos, los primeros permiten medir resultados y magnitudes positivas mediante indicadores derivados de variables empíricas, los segundos son parte de la cosmovisión o del decisor y deriva de sus particulares fines y propósitos (4).

1 Doctor (c) en Ingeniería, Magíster en Administración, Ingeniero Industrial, Economista (b). Profesor en la Facultad de Ingeniería Industrial-UNMSM.

Email: aacevedo@speedy.com.pe

2 Magíster en Dirección de Empresas, Ingeniero Industrial, estudios de Derecho. Profesora en UPG Facultad de Ingeniería de Sistemas e Informática UNMSM.

Email:klinares@speedy.com.pe 
El diseño epistemológico hegeliano considera que los decisores poseen diferentes definiciones del problema, según sus particulares supuestos, valores y paradigmas, esto genera conflicto y confrontación entre diferentes puntos de vista, y la solución se obtiene mediante consenso. A partir de la concepción de sistemas, se considera que no se resuelven problemas aislados sino se manejan situaciones problemáticas o escenarios, donde la resolución de un tema conlleva la aparición de efectos emergentes (deseados y no deseados, esperados y emergentes) (15).

\section{LOS CRITERIOS PARA TOMAR DECISIONES}

Los criterios para la toma de decisiones, objetivos y subjetivos, pueden ser visibles u ocultos, indistintamente, y permiten entender la perspectiva de los decisores y la sistematización del método de aná- lisis y solución, facilitando la posterior implantacion de soluciones.

\section{Marco conceptual de los criterios de decisión}

Los criterios son las bases que se emplean para determinar la mejor decisión, según el contexto. Las decisiones complejas requieren de criterios muy variados. Una solución que minimiza el costo o maximiza la utilidad estará guiada por el criterio costo-beneficio, pero el criterio de relaciones laborales y nivel de empleo modifica la definición de lo que es mejor decisión.

La figura 1 muestra el modelo de decisiones de Buffa, donde el criterio de elección es el tamiz y elemento clave para seleccionar el mejor curso de acción. Este modelo se sustenta en la teoría general de sistemas, aplicada a la gerencia de los sistemas productivos.

Figura 1. Los criterios para la toma de decisiones

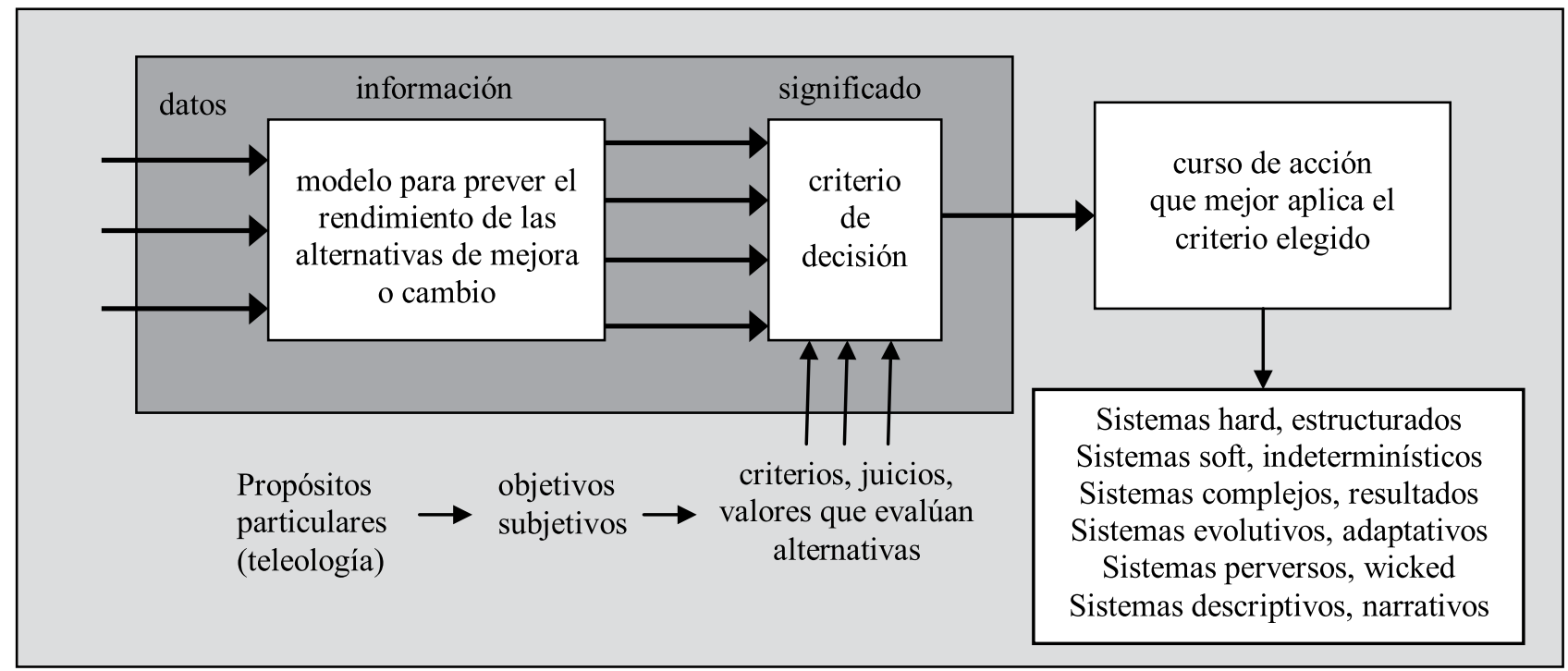

Fuente: Elaboración propia en base a modelo de decisión de Buffa

\section{El rol y criterio del ingeniero industrial}

El rol del ingeniero industrial, en su tarea empresarial de resolución de problemas, es tomar decisiones para la supervivencia y el desarrollo de la organización, dada la complejidad de los sistemas en los que actúa, se ha de contemplar una amplia gama de criterios, complementarios o contradictorios entre sí. Como ejemplo, el criterio costo-utilidad se emplea para decisiones de fabricar o comprar (13).

Toda decisión afecta a diferentes dimensiones de la empresa, por lo que el criterio de decisión delimita el ámbito de solución, ya que no es posible incorporar todas las variables afectadas o que afectan los resultados de la acción. Interpretando el arte tomar, la elección de uno u otro criterio, es pertinente, en función a la perspectiva que asume el decisor y está sujeta a intercambios y concesiones entre decisores (7).

\section{La toma de decisiones para resolver problemas}

La toma de decisiones para resolver problemas empresariales es una de las tareas principales del ingeniero industrial dentro de las organizaciones (9). En su rol decisor, el ingeniero industrial toma 
decisiones bajo diferentes premisas, como mantener la estabilidad del conjunto, orientar el cambio planeado, aprovechar oportunidades emergentes del entorno o diseñar los planes para el futuro.

De acuerdo al enfoque sistémico en operaciones, uno de los elementos más importantes y determinante de la elección es el criterio de decisión que asumen los decisores, cuya responsabilidad es elegir las opciones de transformación para brindar productos con utilidad, cubriendo las necesidades del personal y la comunidad.

\section{LOS CRITERIOS DE DECISIÓN EMPRESA- RIAL}

El criterio adecuado permite hacer las cosas bien, hacer las cosas correctas, y efectuar el cambio para adaptarse y crecer, dentro de la situación problemática que constituye la circunstancia o escenario, donde la medida del éxito es la adaptación al medio, dominio del entorno y el logro de utilidades (3).

\section{Requisitos de los criterios de decisión}

Los criterios de decisión, al desarrollarse, están compuestos por un esquema de indicadores o variables, cuya elección ha de cumplir los siguientes requisitos:

- El criterio mantiene coherencia con el objetivo a alcanzar, derivado de la racionalidad y la consistencia del conocimiento aplicado.
- El criterio de decisión debe ser concordante con las creencias, paradigmas y prejuicios de los decisores.

- La asignación de prioridad (cuantificación del peso de los indicadores) debe ser consistente con la importancia que el grupo asigna a dicho indicador.

- El criterio debe llevar a decisiones cuya aplicación sea posible, dentro del contexto de la situación problemática.

- Las reglas de decisión deben derivar del conocimiento y comprensión de todos los involucrados.

- Las reglas de decisión deben derivar del consenso de los decisores.

- Las premisas y definición del problema deben ser comprendidos y aceptados por los decisores.

- El criterio de decisión ha de ser consecuente con el rol del decisor, el nivel de decisión y con el campo o escenario en que actúa (11).

\section{Los criterios y el rol del decisor}

Tomando como base el estudio del modelo 4D o de las cuatro dimensiones de la empresa y los campos de circunstancia, cuyo antecedente es el modelo sociotécnico (2) y los elementos de ingeniería de sistemas (5), se consideran cuatro tipos de macrocriterios decisionales, relacionados al escenario y a la perspectiva del decisor, se muestran en la tabla 1.

Tabla 1. Criterios de decisión en función al rol del decisor

\begin{tabular}{|c|c|c|c|}
\hline Escenario & Tarea del decisor & Rol que asume & Criterio aplicable \\
\hline $\begin{array}{l}\text { Campo 1. Ambiente ante el grupo humano } \\
\text { Control de recursos y personas. }\end{array}$ & $\begin{array}{l}\text { Propia naturaleza } \\
\text { Sophia, Politik, } \\
\text { Poetik }\end{array}$ & $\begin{array}{l}\text { Académico / } \\
\text { político }\end{array}$ & $\begin{array}{l}\text { Posición dentro } \\
\text { de una jerarquía }\end{array}$ \\
\hline $\begin{array}{l}\text { Campo 2. Ambiente en sectores y organizaciones } \\
\text { Productivo y económico }\end{array}$ & $\begin{array}{l}\text { Productivo } \\
\text { Poiesis } \\
\text { Apolo y Dionisio }\end{array}$ & $\begin{array}{l}\text { productivo / } \\
\text { entretenimiento }\end{array}$ & $\begin{array}{l}\text { Optimización } \\
\text { dentro de las } \\
\text { condiciones } \\
\text { reales } \\
\end{array}$ \\
\hline $\begin{array}{l}\text { Campo 3. Las fuerzas de cambio en los individuos } \\
\text { Persona y ambiente humano. }\end{array}$ & $\begin{array}{c}\text { Social } \\
\text { Teleológica, } \\
\text { comu-nicativa, } \\
\text { normativa, } \\
\text { dramatúrgica }\end{array}$ & $\begin{array}{l}\text { Principista / } \\
\text { pragmático }\end{array}$ & $\begin{array}{l}\text { Satisfacción y } \\
\text { bienestar de } \\
\text { grupo e individuos }\end{array}$ \\
\hline $\begin{array}{l}\text { Campo 4. Fuerzas de cambio en sectores y } \\
\text { empresa } \\
\text { Proyección, predicción y desarrollo. }\end{array}$ & $\begin{array}{l}\text { Praxis } \\
\text { Devenir, } \\
\text { desarrollo, } \\
\text { evolución, } \\
\text { revolución }\end{array}$ & $\begin{array}{l}\text { Planificador / } \\
\text { Oportunista }\end{array}$ & $\begin{array}{l}\text { Oportunidad ante } \\
\text { el cambio y futuro }\end{array}$ \\
\hline
\end{tabular}

Fuente: Elaboración propia 
Estos criterios (1) pueden ser:

a) De posición, basados en el control y el poder de recursos y personas.

b) De optimización, basados en el manejo de recursos para obtener resultados, es la acción en el mundo real.

c) De satisfacción, basados en el grupo, la pertenencia, la regulación social de la conducta.

d) De oportunidad, basados en proyecciones, predicciones y contingencias.

\section{El modelo conceptual de los criterios de decisión}

Mediante la metodología de diseño de modelos de sistemas suaves (6), se han elaborado los seis grandes criterios que, a modo de paraguas, contienen las posibilidades que se aplican para orientar las decisiones referidas a resolver problemas en diferentes niveles de resolución, principalmente estratégicos (12). Ver la figura 2.

Los criterios objetivos se sustentan por la razón y se refieren a las decisiones racionales orientadas a la acción. Son las que buscan el logro de una o más metas concretas y verificables, conllevando la modificación de condiciones, eventos, causas y efectos.

Para la empresa se consideran cuatro tipos de criterios ( 1 al 4), relacionados al escenario en que se ubica y al rol que el decisor asume en dicho escenario.

Figura 2. Principales o grandes criterios de decisión y enfoque del ingeniero industrial como decisor

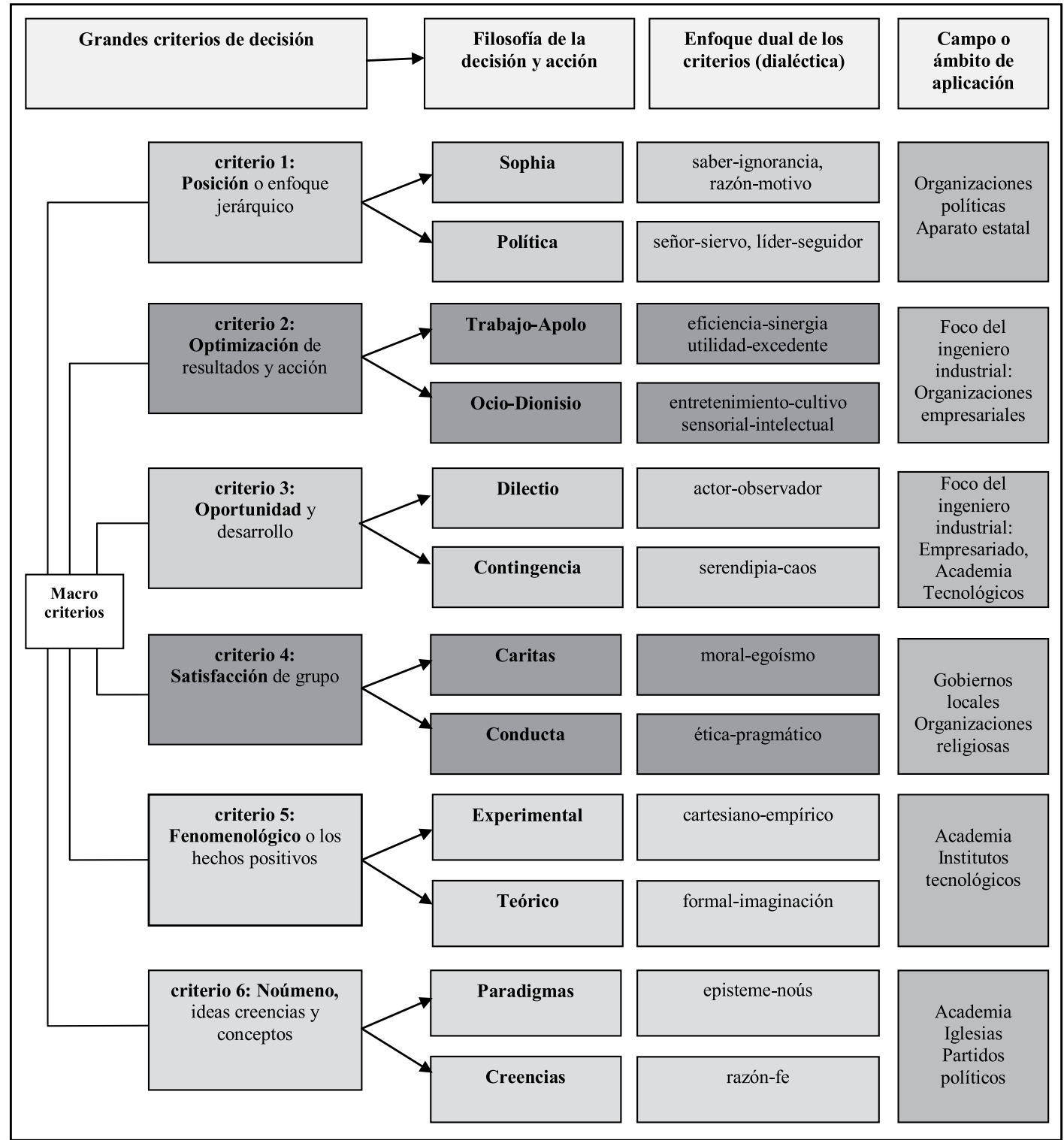

Fuente: Elaboración propia 


\section{ESTUDIO SOBRE LOS CRITERIOS DE DECI- SIÓN}

El ingeniero industrial, en su papel de resolución de problemas, afronta las situaciones problemáticas bajo criterios delimitados por el paradigma de la administración científica y el paradigma de sistemas

\section{Objetivo, problema de investigación e hipótesis}

El ingeniero industrial, en sus tareas de gestión en organizaciones, se encuentra enmarcado por la posición que ocupa y el rol que asume dentro del escenario donde se aplica la decisión. El criterio se enmarca por el propósito del que asume la decisión.

La pregunta de investigación sería: ¿Cómo es el criterio pertinente para delimitar la elección del directivo, entre un rango de opciones de decisión?

La hipótesis general $(\mathrm{H} 0)$ es "La toma de decisiones en el mundo de la empresa considera que los criterios empleados ejercen igual influencia en la elección de la alternativa de resolucion del problema.", y la hipótesis alterna (H1) es "La toma de decisiones en el mundo de la empresa considera que los criterios empleados ejercen influencia diferente en la elección de la alternativa de resolucion del problema".

Los objetivos específicos son:

- Definir, exploratoriamente, los criterios aplicables por los directivo para la toma de decisiones en las organizaciones empresariales.

- Identificar, exploratoriamente si los criterios de decisión afectan la elección directiva.

- Identificar la orientación hacia algún criterio de decisión por los directivos de empresa.

- Plantear, exploratoriamente, la relación entre los campos de circunstancia y los tipos de criterios de decisión.

\section{Diseño de experimento, instrumento de recolec-} ción de información y muestra

A partir de la hipótesis, se definió la matriz básica de datos que facilitó la elaboración una herramienta de recolección de datos, con diez opciones de decisión y con seis alternativas de respuesta. Mediante estadística inferencial, se definieron dos muestras aleatorias, cada una de 33 profesionales de empresa. La primera muestra en Lima conformada por ingenieros industriales e ingenieros de sistemas, la segunda muestra en provincias conformada por ingenieros industriales en su mayoría, administradores y economistas, con estudios de postgrado.

Se realizaron talleres de trabajo donde se aplicó el cuestionario, se analizó con software de computa- dora y se discutieron los hallazgos en los talleres de trabajo, con los profesionales encuestados. Previamente, se desarrollaron entrevistas presenciales con académicos y directivos para identificar el modelo conceptual de los criterios de decisión, se desarrollaron y discutieron casos-estudio para la resolución de problemas en diferentes escenarios cuyos resultados preliminares, referido a el planteamiento de diferentes criterios indicaron elementos de decisión que requieren futuros estudios, específicos y a profundidad.

Para resolver la pregunta del problema de investigación, se elabora un diseño aleatorio (8), a modo de experimento donde se comparan $\mathrm{k}$ tratamientos del factor $C$ (criterios). Este diseño se denomina ANOVA o análisis de varianza de un factor, que compara las medias de los criterios. Los datos detallados, obtenidos de las muestras, no se incluyen en el presente trabajo, por razones de espacio.

Se emplea el modelo de regresión lineal y prueba de hipótesis mediante análisis de varianza, considerando:

$\mu=$ media total

Error o residuo: variables aleatorias que se desvían de media,

independientes y normales, de la forma normal $\mathrm{N}$ $(0$, oi2)

$\varepsilon i j=X i j-\mu i$

entonces: $\quad X i j=\mu i+\varepsilon i j, \quad \forall i=1,2,3,4,5,6$; $\mathrm{j}=1,2, \ldots 33$

\section{Efecto del tratamiento i:}

$\alpha i=\mu \mathrm{i}-\mu$

donde:

$$
\sum_{i=1}^{k} \alpha_{i}=0
$$

El modelo de un factor completamente aleatorio:

$$
X_{\mathrm{ij}}=\mu_{\mathrm{i}}+\boldsymbol{\alpha}_{\mathrm{i}}+\varepsilon_{\mathrm{ij}}
$$

\section{$\left.1^{\circ}\right)$ Hipótesis}

H0 : $\mu 1=\mu 2=\mu 3=\mu 4=\mu 5=\mu 6=\mu$

Todas las $\mu \mathrm{i}$ son iguales; donde $\mathrm{i}=1,2, \ldots \mathrm{k}, \mathrm{k}=6$

ó: no hay efecto de $\mathrm{k}$ tratamientos 
H1: No todas las $\mu$ i son iguales; donde $i=1,2,3$, $4,5,6$

$$
\text { ó: } \quad \exists i / \alpha_{i}=0
$$

\section{Donde:}

$\mu \mathrm{i}$ : Media de las elecciones por el tratamiento i $\left.2^{\circ}\right)$ Nivel de significancia (n.s.) y Nivel de confianza (n.c.)

$$
\begin{aligned}
& \text { Nivel de significancia } \alpha=.05 \\
& \text { Nivel de confianza } 1-\alpha=.95
\end{aligned}
$$

\section{$\left.3^{\circ}\right)$ Se calcula F. Cálculo mediante SPSS}

Los resultados resumen para ambas muestras se presentan en las tablas 3 y 4 , respectivamente.

Tabla 2. Estudio ANOVA -Lima

\section{ANOVA}

preferencia

\begin{tabular}{|l|c|c|c|c|c|}
\hline & Suma de cuadrados & gl & Media cuadrática & F & Sig. \\
\hline Inter-grupos & 48051,576 & 5 & 9610,315 & 139,507 &, 000 \\
\hline Intra-grupos & 13226,424 & 192 & 68,888 & & \\
\hline Total & 61278,000 & 197 & & & \\
\hline
\end{tabular}

Tabla 3. Estudio ANOVA -Provincias

ANOVA

preferencia

\begin{tabular}{|l|c|c|c|c|c|}
\hline & Suma de cuadrados & gl & Media cuadrática & F & Sig. \\
\hline Inter-grupos & 43968,652 & 5 & 8793,730 & 118,515 &, 000 \\
\hline Intra-grupos & 14246,303 & 192 & 74,199 & & \\
\hline Total & 58214,955 & 197 & & & \\
\hline
\end{tabular}

\section{$\left.4^{\circ}\right)$ Prueba $F$ para análisis de varianza de un factor:}

Gráficamente, se presenta en la figura 3.

Figura 3. Rechazo de la hipótesis principal para muestra 1-Lima y muestra 2-Provincias

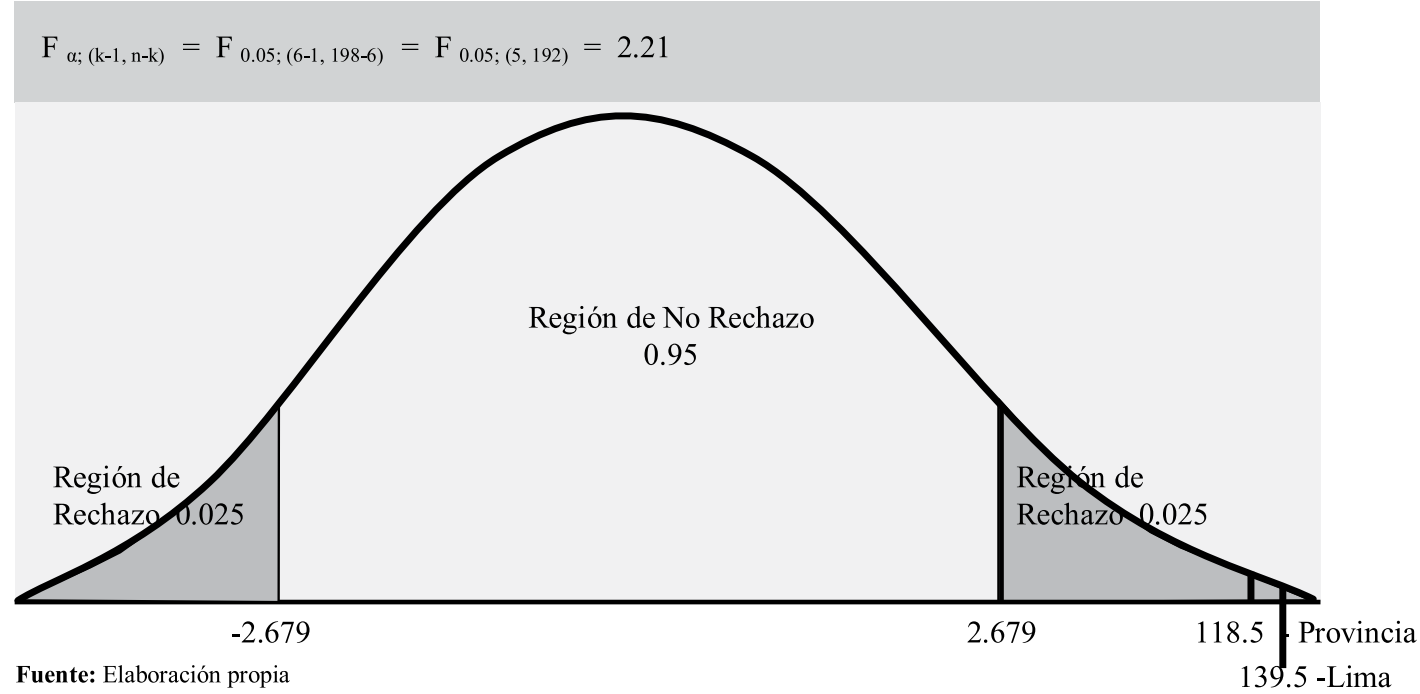




\section{$\left.5^{\circ}\right)$ Análisis y decisión en la muestra}

Para la muestra 1 con ingenieros industriales de Lima y para la muestra 2 con ingenieros industriales y administradores de Provincias, se obtienen resultados semejantes.

Entonces, para un nivel de significación de .05 el $F$ calculado es superior al $F$ teórico, lo que señala que son parte de una población con criterios semejantes.

Por lo tanto, se rechaza la hipótesis general que afirma que los criterios empleados en la toma de decisiones en el mundo de la empresa ejercen igual influencia en la elección de la alternativa de resolucion de problemas.

De igual manera, no se puede rechazar la hipótesis alterna que indica que los criterios empleados ejer- cen influencia diferente y relevante en la elección de la alternativa de resolucion de problemas.

\section{Análisis y discusión de los resultados}

El programa computarizado desarrollado considera el análisis exhaustivo de los resultados, los que se analizaron desde diferentes perspectivas.

Los resultados del estudio muestran que los criterios que se emplean para tomar decisiones no son uniformes. La figura 4 referida a la orientación de los decisores en un eje cartesiano, muestra una marcada preferencia hacia el campo 2 , que correspondería al criterio de optimización o la búsqueda del resultado, en el campo de recursos y empresa. Los decisores presentan mayor concentración en los campos relacionados a la administración de recursos y la operatividad.

Figura 4. Resultados general del estudio- El criterio de decisión prevaleciente

Muestra 1 - Lima

3a. coordenadas de crit $\cdot$ series 1

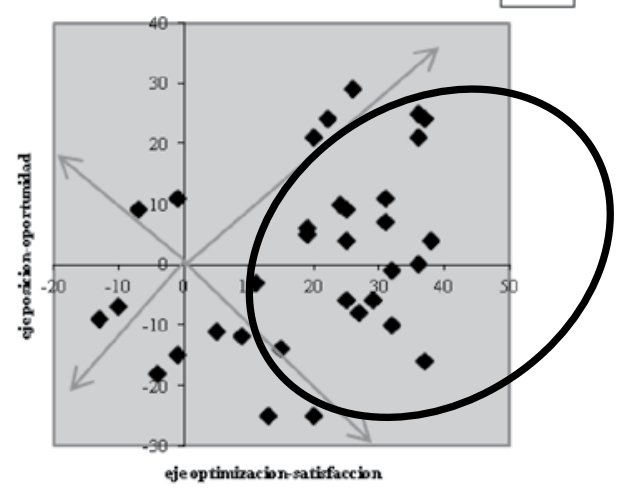

Fuente: Elaboración propia

El estudio muestra que los decisores se orientan, marcadamente, a la economía de los recursos y a la funcionalidad de los procesos, esto significa una fuerte priorización de los asuntos de trabajo y utilitarismo. En forma extrema se observa, en los resultados, que los aspectos relacionados a personas, como la conciencia de grupo (ética y respeto) y al conocimiento (saber sistematizado) no tienen importancia para el decisor.

Básicamente, los decisores de ambas muestras, otorgan un $61 \%$ y $79 \%$ a los criterios económicos y empresariales. A pesar que los participantes se ubican en posiciones de decisiones directivas, los criterios de posición, poder y mando no son los relevantes, solo representan un $9 \%$ de prioridad, en ambas muestras.
Muestra 2 - Provincia

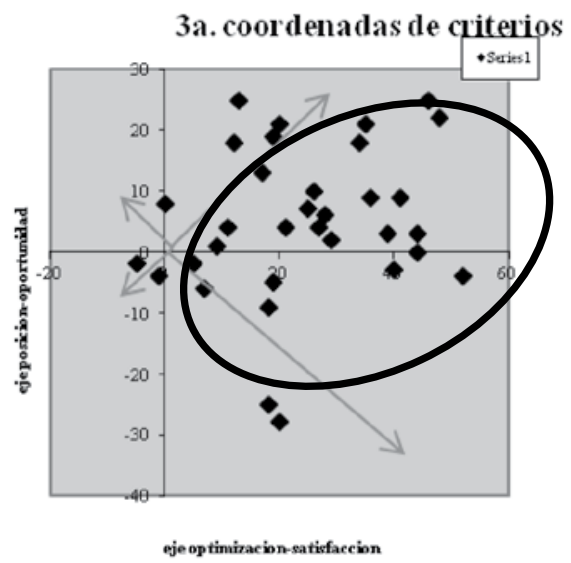

\section{CONCLUSIONES}

En el presente estudio sobre los criterios decisionales, se ha empleado análisis estadístico inferencial mediante de diferencia de medias, por el cual se ha determinado que se rechaza la hipótesis general y se acepta la hipótesis alternativa, por lo que, los decisores plantean sus elecciones según el criterio pertinente, el que está de acuerdo con la circunstancia o contexto, el escenario que enfrenta y el rol que ha asumido en dicho contexto. Por otro lado, no es posible determinar si los decisores son consecuentes con sus preferencias personales. En las muestras de Lima y provincias, los decisores enfatizan los criterios enfocados en procesos y estructuras ( $61 \%$ y $79 \%$, respectivamente), por encima de los criterios sobre personas y cambio, que presen- 
tan menor importancia (6\% y $3 \%$ respectivamente).

Los resultados del presente estudio exploratorio, referido al efecto de los criterios para la toma de decisiones, permiten resumir las siguientes conclusiones:

1. Los decisores dentro de organizaciones, presentan perfiles de criterio para la elección, semejantes entre sí, lo que indica que no se generan conflictos en la elección de opciones.

2. Los decisores otorgan mayor prioridad a los aspectos económicos de la decisión, de manera que es previsible cierto sesgo hacia la eficiencia (de recursos) y sinergias (de funcionamiento) sobre la satisfacción o motivación del personal.

3. El criterio político o de poder manifiesta menor prioridad que los criterios de eficiencia, lo que se explica por el foco de decisión de los participantes de la muestra.

4. Los criterios relacionados a personas (bienestar), presentan menor importancia, lo que es una cuestión a plantear en estudios poesteriores, ya que requiere mayor estudio.

5. Los logros inmediatos presentan mayor prioridad, respecto a las previsiones, así, las técnicas contingentes son más empleadas que las herramientas de planeamiento prospectivo.

\section{REFERENCIAS}

[1] Acevedo, Adolfo, (2010). "El modelo conceptual de las 4 Dimensiones para la resolución de problemas". Industrial Data Revista de Investigación, Vol. 13, N², julio-diciembre.

[2] Acevedo, Adolfo, (2003). "El modelo de los sistemas sociotécnicos en la teoría y praxis empresarial", Proceedings XXXVIII Asamblea Anual CLADEA. Perú.
[3] Ackoff, Russell (2000). Recreación de las corporaciones. Un diseño organizacional para el siglo XXI. Ed. Oxford University Press. México.

[4] Buffa, Elwood (1981). Administración de Operaciones. La administración de sistemas productivos. Ed. LIMUSA.

[5] Blair \& Whitston (1973). "Elementos de Ingeniería de Sistemas Industriales". Editorial Prentice-Hall Internacional. España.

[6] Checkland \& Scholes (1994). La Metodología de los Sistemas Suaves de Acción. Noriega Editores. México.

[7] Cooper, Joseph (1961). The Art of Decision Making. Ed. Doubleday \& Company, Inc. New York.

[8] Hernández,Sampieri, Fernández, Baptista (2010). Metodología de la investigación. Quinta edición. Ed. Mcgraw-Hill, Chile.

[9] Kast \& Rosenzweig (1968). Administración en las organizaciones, enfoque de sistemas y de contingencias. Ed. Mcgraw-Hill, México.

[10] Marías, Julián (1974). Historia de la Filosofía. Ed. Revista de Occidente. España.

[11] McGuire, Joseph (1974). Teorías del comportamiento empresario. Ed. El Ateneo. Buenos Aires.

[12] Mintzberg, Quinn y Voyer (1997). El proceso estratégico. Conceptos, contextos y casos. Prentice Hall. México.

[13] Monks, Joseph (1985). Operations Management. 2da. Ed. Mcgraw-Hill, USA.

[14] Navarro y Calvo (1990). Historia de la Filosofía. Ed. Anaya, España.

[15] Zandin, Kjell (2005). Maynard Manual del Ingeniero Industrial. 5ta. edición. Ed. McgrawHill, México. 